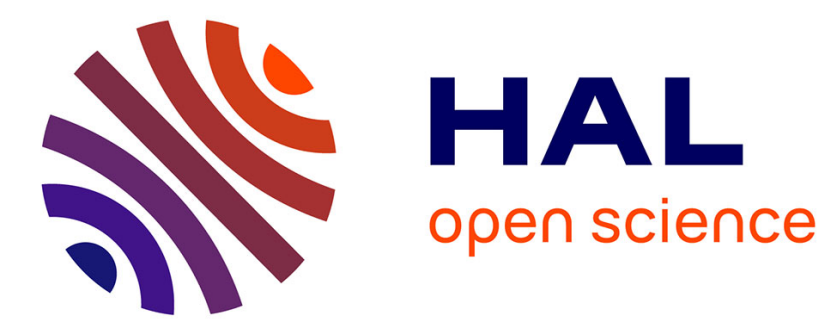

\title{
Adaptive Learning Organizer for Web-Based Education
}

Amel Yessad, Catherine Faron Zucker, Med Tayeb Laskri, Rose Dieng-Kuntz

\section{To cite this version:}

Amel Yessad, Catherine Faron Zucker, Med Tayeb Laskri, Rose Dieng-Kuntz. Adaptive Learning Organizer for Web-Based Education. International Journal of Web-Based Learning and Teaching Technologies, 2008, 3 (4), pp.57-73. 10.4018/jwltt.2008100103 . hal-03503176

\section{HAL Id: hal-03503176 https://hal.inria.fr/hal-03503176}

Submitted on 27 Dec 2021

HAL is a multi-disciplinary open access archive for the deposit and dissemination of scientific research documents, whether they are published or not. The documents may come from teaching and research institutions in France or abroad, or from public or private research centers.
L'archive ouverte pluridisciplinaire HAL, est destinée au dépôt et à la diffusion de documents scientifiques de niveau recherche, publiés ou non, émanant des établissements d'enseignement et de recherche français ou étrangers, des laboratoires publics ou privés. 


\section{Adaptive Learning Organizer for Web-based Education}

Amel Yessad, Edelweiss, INRIA Sophia Antipolis, 2004 route des Lucioles, BP 93, 06902 Sophia Antipolis, France and Université 20 Août 1955, BP 26 El Hadaik, 21000 Skikda,

Algérie

Amel.Yessad@inria.fr, Tel: +33 497155302, fax: +33 492387783

Catherine Faron-Zucker, Kewi, I3S, Université de Nice Sophia, CNRS, 930 route des Colles, BP 145, 06930 Sophia Antipolis cedex, France faron@polytech.unice.fr, Tel: +33492965190

Rose Dieng-Kuntz, Edelweiss, INRIA Sophia Antipolis, 2004 route des Lucioles, BP 93, 06902 Sophia Antipolis, France

Rose.Dieng@inria.fr, Tel:+33 492387810, fax: +33 492387783

Med Tayeb Laskri, GRIA-LRI, Université Badji Mokhtar, BP 12, 23000 Annaba, Algérie laskri@univ-annaba.org,

Tel: +213 38872678, fax: +21338 872436 


\title{
Adaptive Learning Organizer for Web-based Education
}

\begin{abstract}
Adaptive learning support for learners becomes very important in the context of increasing re-use of resources from heterogeneous and distributed learning repositories. This paper presents OrPAF, an Adaptive Educational Hypermedia (AEHS) and web-based System which integrates semantic web models and technologies in order to achieve interoperability with e-learning systems. The key feature of OrPAF is the construction of adaptive hypermedia courses: both the course structure and the course content are dynamically generated and adapted to learners. On the one hand, a learning ontology is proposed to describe, at a meta-level, abstract characteristics of an e-learning system. This learning ontology is instantiated to construct learning models: domain model, learner model and pedagogical model. On the other hand, semantic annotations and a semantic relevance measure are proposed to improve the LOM metadata associated to learning resources in order to reuse and share them. We experimented the realized prototype on learners in order to evaluate the usability of OrPAF and conceptual capabilities developed by learners who used it.
\end{abstract}

Keywords: AEHS, SW-EL, Ontology, Learning resource metadata.

\section{INTRODUCTION}

Many researches propose personalized learning supports in order to reuse and share learning resources from distributed repositories (Nejdl et al., 2002; Miklos et al., 2003; Dolog et al., 2004). The personalization of existing learning resources can be a solution to the problem of developing online courses. However, the personalized learning that used distributed metadata of learning resources is still an unsolved problem in the e-Learning research area.

Considering the increasing re-use of learning resources from the Web it becomes almost impossible for the learners, experts and formation responsible to get an overview of all the available information relevant to their current needs, tasks, roles and goals. And even if they find some materials, which seem suitable, they are not able to assess completely whether the found content is entirely appropriate for their goals or current knowledge and cognitive state. For that reason, learning resources searched from web repositories must be first subject to a pedagogy engineering work in order to give them reusable in the context of a specific training for specific learners. This engineering work is time and effort consuming in the design step of an e-learning system. To solve this problem, we propose an approach that moves part of this engineering effort from the training responsible/ expert to the software system and delivers an adaptive hypermedia course directly to learners. 
In this context we aim to offer personalized course support which generates dynamically, for each learner, an individualized course structure and an individualized course content by selecting the most optimal learning concepts (e.g. the concept Function in the Algorithmic and Programming Languages domain) and the most relevant learning web resources (e.g. the Definition of the concept Function) at any moment. Optimal learning concepts and associated relevant learning resources are selected to bring the learner closest to his/her ultimate learning goal. This approach is well suited for individual and autonomous learners taking a self-study distance-learning course. They can be employees in an organization who have various experiences and background knowledge and where employees evolve in competitive economic environment and require longlife learning.

We propose a learning organizer which generates adaptive hypermedia courses and reuses learning resources from distant web repositories, called « Organisateur de Parcours Adaptatifs de Formation»(OrPAF). Queried learning resources are already annotated with LOM metadata but stay very difficult to reuse automatically because of the semantic lack of LOM metadata. Our work is based on semantic web models, particularly ontologies and semantic annotations, in order to improve the quality of LOM metadata and describe in a standardized way several characteristics of e-learning system (e.g. learning resource, pedagogical strategy, learner model). The semantic Web for E-Learning (SW-EL) field has shown the greatest activity in this trend with several interesting and recurring practices (Dolog et al., 2003; Aroyo et al., 2004; Yessad et al., 2006).

Our aim is to improve the learning process efficiency (1) by providing the learner with adaptive learning paths according to his/her level of knowledge, learning goal and time constraints; and (2) by reusing learning resources of different web repositories. On the one hand, an adaptive learning path is constructed on the base of the conceptual structure of the domain model (e.g. the Algorithmic and Programming Languages domain) and the learner model (e.g. beginner). In the learning organizer, the learner is assisted to construct a «correct» representation of a particular domain of knowledge and the learning is self regulated (Pintrich et al., 2002; Perry et al., 2004). For this purpose, we apply filters on the domain model to generate a map of relevant learning concepts. We call this map Adaptive Cognitive Map (ACM). An ACM is automatically generated and displayed to a learner, which takes into account a specific goal, the knowledge and temporal constraints of the learner. An ACM represents an adapted view of the structure of the hypermedia course. On the other hand, learning resources that are queried from distant web repositories like ARIADNE or created locally by domain experts are annotated by adding a conceptual layer on LOM metadata description layer. We propose a measure to evaluate the semantic relevance of annotated learning resources in order to associate them to the generated ACM and recommend them to the learner.

This paper is organized as follows: section 2 presents an overview and a positioning of our learning organizer approach. Section 3 shows how we represent e-learning knowledge in a meta-model and how we use it to construct different e-learning models. We explain in section 4 the generation of an adaptive structure and an adaptive content of the hypermedia course. Section 5 is dedicated the implementation of OrPAF and our evaluation protocol. 


\section{RELATED WORK}

Representative examples of personalized support for learners are adaptive textbooks constructed with AHA! (De Bra et al., 2003), InterBook (Brusilovsky et al., 1998) and NetCoach (Weber et al., 2001), or adaptive courses within ELM-ART (Brusilovsky et al., 1996), PAT (Ritter, 1997) and AIMS (Aroyo et al., 2001). There are also more global but still highly specialized efforts, such as ARIADNE and EdNa courseware-reusability frameworks that provide repositories of reusable learning resources. In this context, our research aims to propose a learning organizer which is an adaptive educational hypermedia and web-based system (AEHS). Our learning organizer integrates semantic web technologies like ontologies, semantic annotation and learning standards in order to achieve interoperability with e-learning systems.

Similarly to the Dynamic Course Generation system (Brusilovsky et al., 2003) and researches of (Ullrich, 2004) and (Dehors et al., 2006), the core of our learning organizer is the explicit representation of the domain model, separated from learning resources and pedagogical strategies. We define a learning ontology that describes characteristics of elearning systems (e.g. learner, pedagogical activity). This learning ontology is a meta-model which describes abstract learning characteristic independent of a specific learner, a specific domain (e.g. the Algorithmic and Programming Languages domain), a specific pedagogical strategy (e.g. the deductive strategy) or a specific learning resource (e.g. Slides created by JamesRumbaugh introduce the Object Modelling notion). The meta-model is instantiated to construct specific learning models: the domain model, the learner model and the pedagogical model. Contrary to the learning ontology, these models describe respectively learning concepts of a specific domain (e.g. Arithmetic Operators in the Algorithmic and Programming Languages domain), a specific learner and a specific pedagogical strategy (e.g. inductive strategy). In contrast with other approaches mentioned above, by the addition of learning characteristics at the meta-model makes easier the extensibility of our learning system.

In authoring tools like InterBook (Brusilovsky et al., 1998), MetaLinks (Murray, 2001), and NetCoach (Weber et al., 2001), the expert explicitly provides the course structure as a textbook hierarchy (like page1 has subsection page1-1). In contrast, in our approach, the structure of the course is an Adaptive Cognitive Map (ACM), i.e. a sub-graph of the graph of concepts which represents the domain model. In fact, we use the structure of the domain model as a roadmap to generate course paths. Given a certain goal concept (e.g. Arithmetic Operator) that the learner wants to acquire and given his/her learner model, our learning organizer generates a map of learning concepts to the learner in order to achieve his/her goal.

Moreover, our learning organizer implements a query component which uses a measure of the relevance of a particular learning resource for the learning context of the learner. Many researches propose to query resources from the web by relaxing the concepts of the query to other concepts close to them in the domain model. Close concepts are identified by using distance measures. In all these researches (Corby et al., 2004; Zhong et al., 2002; Rada et al., 1989; Resnik, 1995; Wu et al., 1994), the distance measure is based on types of learning concepts in the domain model where the distance between concepts is always a constant 
value. The originality of our approach stands in the use of a relative weight-based relevance measure. Weights of learning concepts depend of the learning context and are not fixed in advance.

\section{LEARNING ORGANIZER KNOWLEDGE}

Because of the increasing complexity and heterogeneity of knowledge in e-learning systems (e.g. domain knowledge, learner knowledge, pedagogical knowledge), we require an efficient and modular knowledge organization. We represent our learning organizer knowledge in two levels: meta-model level and model level. The meta-model knowledge is used to construct learning models which are used by functional components of the learning organizer: Querying resource components, annotation component, testing component, and course generation component (cf. fig. 1).

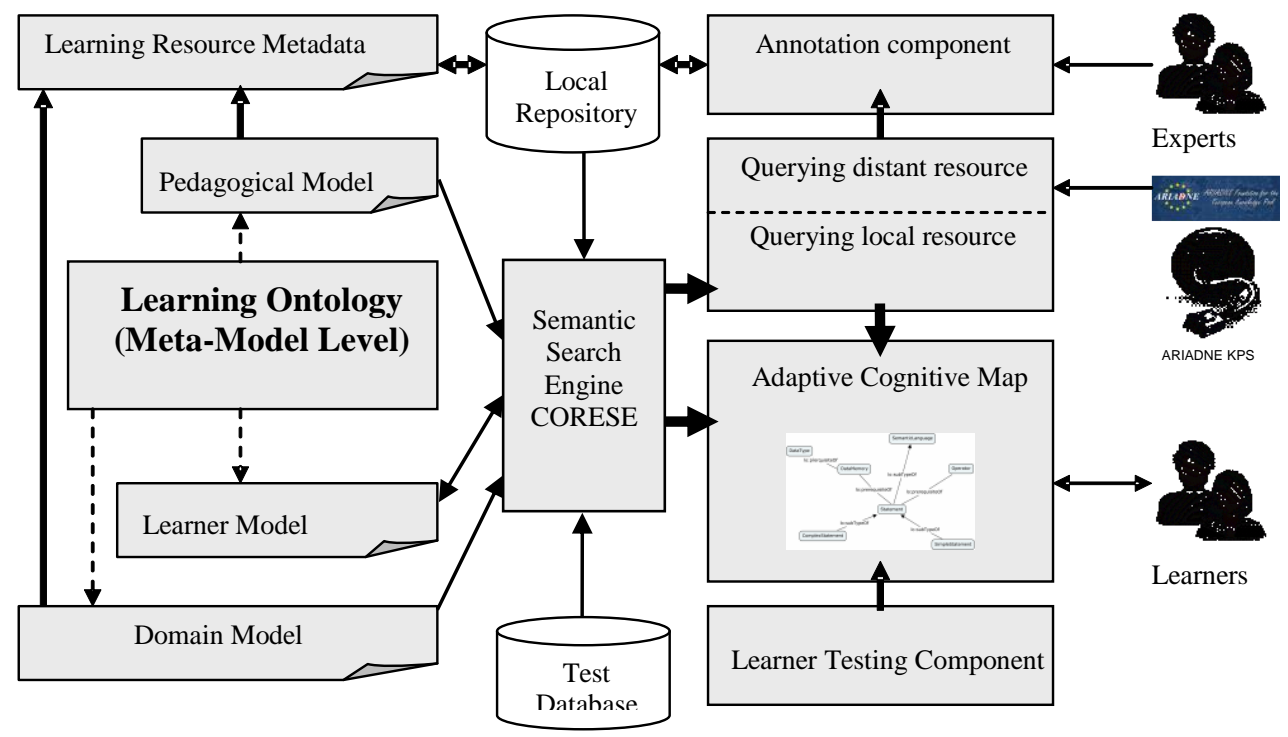

Fig. 1. Architecture of the OrPAF system

\section{Learning Ontology}

The learning ontology we developed is the meta-model and the backbone of the learning process. It describes classes and properties that are instantiated in order to specify the domain of interest (e.g. the Algorithmic and Programming Languages domain), profiles of the learners (e.g. a beginner) and pedagogical strategies (e.g. deductive strategy). Classes and properties are seen as general objects. So, the learning ontology (meta-model) is instantiated to construct three learning models: a domain model, a learner model and a pedagogical model. Contrary to the learning ontology, these models describe specific objects. For instance, in the learning ontology, we describe types of learning concepts of a domain (e.g. a GoalConcept) 
and relationships between these types (e.g. prerequisite Of relationship) whereas in the domain model we describe concrete learning concepts and their relationships. For instance, the concept Operator and the concept Statement are instances of the class LearningConcept defined in the meta-model (cf. fig. 2); and in the domain model, the concept Operator is related to the concept Statement by the relationship prerequisiteOf (cf. fig. 3).

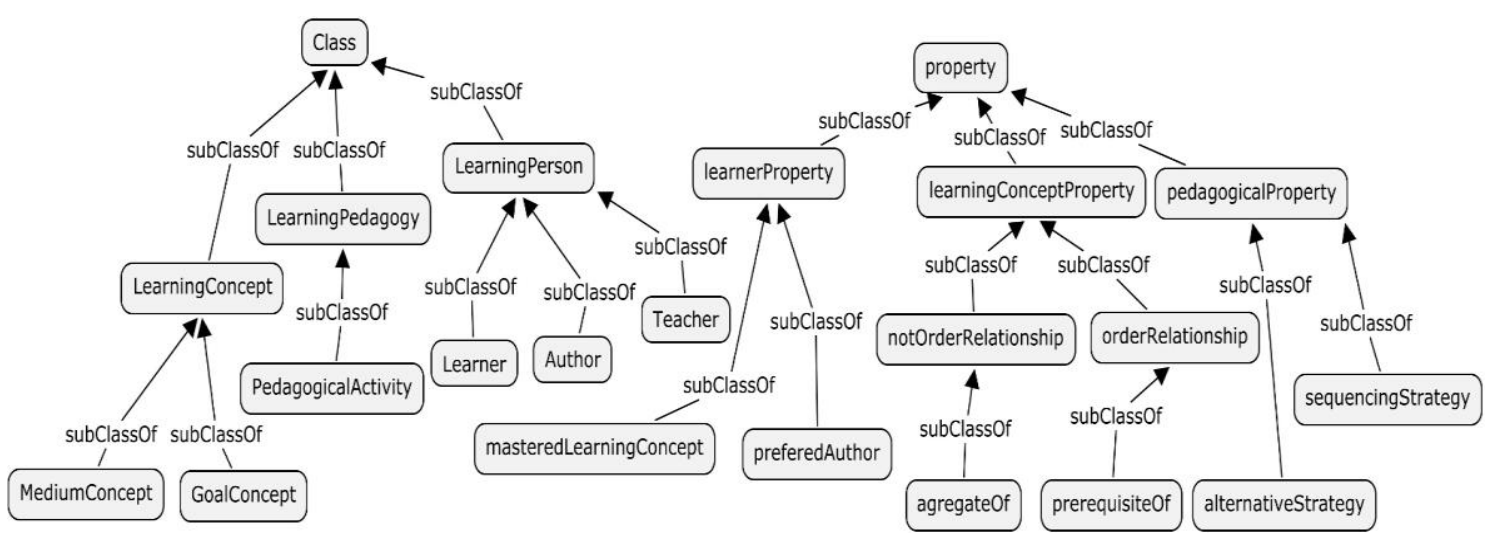

Fig. 2. An excerpt of learning ontology

\section{Learning Models}

The learning ontology is instantiated into three learning models: the domain model, the learner model and the pedagogical model.

\section{Domain Model}

The domain model represents the domain of interest where the learner evolves. A specific domain of interest (e.g. Algorithmic and programming languages) is described by learning concepts and their mutual relationships in a specific discipline. In fig. 3, we show a fragment of the domain knowledge covering learning concepts of «Algorithmic and programming languages »domain, including the subClass $O f$ and the prerequisiteOf relationships between learning concepts.

We build Adaptive Cognitive Map (ACM) by filtering the domain model. An ACM is a sub-graph of the whole graph of related learning concepts. It is used by a learner to construct his/her cognitive representation of the domain of interest in order to achieve his/her learning goal. Learning concepts related with an order relationship (e.g. prerequisiteOf) must be taught following a certain order. 


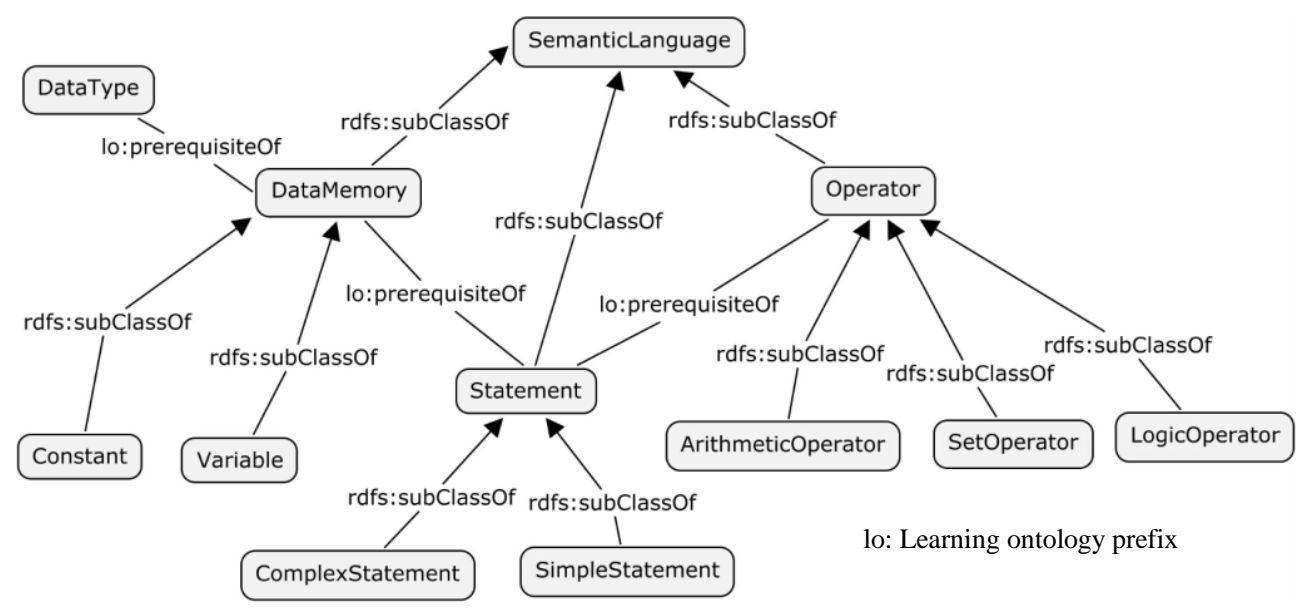

Fig. 3. An excerpt of the domain model «Algorithmic and programming languages »

\section{Learner Model}

The learner model captures knowledge and preferences of the learner. It represents what the system knows about the learner. In our learning organizer, we represent the knowledge of the learner by the overlay model (Galeev et al., 2004). For instance, as shown in fig.4, if the learning concept Operator is mastered by the learner Laura, the knowledge <Laura, masteredLearningConcept, Operator> occurs in the learner model, else the concept Operator is unknown by the learner Laura. The learner model changes during the learning process when the learner passes tests. In this way, our learning organizer provides mechanism for self regulated learning (Pintrich et al., 2002; Perry et al., 2004).

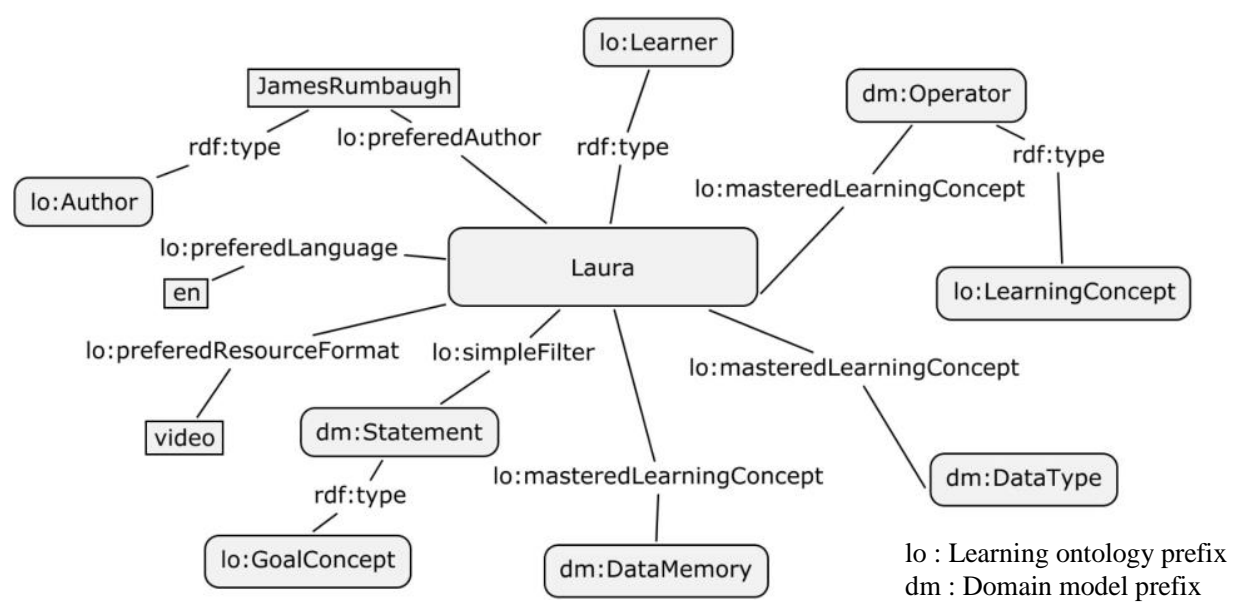

Fig. 4. An excerpt of a learner model 


\section{Pedagogical Model}

The structure of the domain model alone is not sufficient to decide how to present the selected learning concept to the learner, i.e. what pedagogical type of learning resources to select, or how to sequence several learning resources to teach a given learning concept. For this purpose, we define a pedagogical model which describes pedagogical strategies to teach learning concepts. It describes different pedagogical activities (e.g. exercise, lecture) and their relationships. For instance, as shown in fig. 5, the Definition activity (instance of the class PedagogicalActivity in the meta-model) must precede the Exercise activity; both activities are related by the sequencingStrategy property which is defined in the meta-model. The alternativeStrategy relationship between pedagogical activities means that learning resources related to these pedagogical activities can be accessed by a learner in any order whereas the sequencingStrategy relationship requires an order in the presentation of learning resources to the learner.

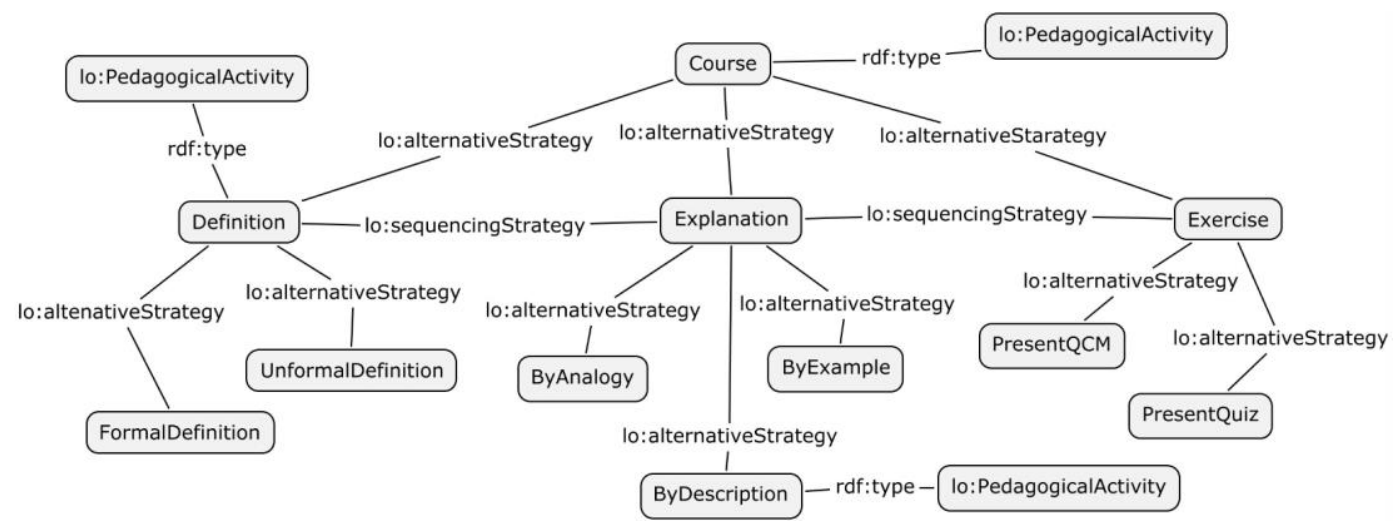

lo : Learning ontology prefix

Fig. 5. An excerpt of the pedagogical model

\section{ADAPTIVE COURSE GENERATION}

The learning organizer aims to construct a hypermedia course as combination of an adaptive course structure and adaptive course content. The course structure is represented by an Adaptive Cognitive Map and the course content is a set of reused relevant learning resources searched from external learning repositories.

\section{Adaptive Cognitive Map (ACM)}

An ACM is a course structure generated and displayed by the learning organizer in order to help the learner to construct a «correct» mental representation of the learning domain. It is a sub-graph of the domain model and contains learning concepts that the learner must learn to 
achieve his goal in required time. An ACM enables the learner to navigate between the learning concepts of the course. We distinguish between three cognitive maps constructed on the goal concept set $G$ of the domain model $(D M)$ : simple $M_{s}(G)$, hierarchical $M_{h}(G)$ and relational $\mathrm{M}_{\mathrm{r}, \mathrm{m}}(\mathrm{G})$. Each one of these maps results from the application of a specific filter on the domain model and only the concepts which pass the filter are displayed to the learner.

- Simple $\mathrm{M}_{\mathrm{s}}(\mathrm{G})$ is the smallest map. It is composed of the goal concept and all concepts related to it directly or by transitive closure of order relationships.

- Hierarchical $\mathrm{M}_{\mathrm{h}}(\mathrm{G})$ is the cognitive map that extends the simple cognitive map to descendants and ascendants of the goal concept.

- Relational $\mathrm{M}_{\mathrm{r}, \mathrm{m}}(\mathrm{G})$ is the cognitive map that extends the simple cognitive map to all concepts related to the goal concept by a path of relationships, the length of this path being less than $\mathrm{m}$.

OrPAF implements each of these three filters. For the same concept goal, the filter depends on learner temporal constraints: the simple filter for learners with hard temporal constraints, the hierarchical filter for learners with medium temporal constraints and the extended filter for learners with flexible or no temporal constraints. This approach can be compared to the micro, the meso and the macro learning approaches (Hug, 2005).

Before displaying the ACM to a learner, an additional adaptation layer is applied on it. It consists of applying rules in order to annotate each learning concept similarly to link annotation technique in adaptive Hypermedia (De Bra et al., 1999). Different icons represent different states of a learning concept (cf. fig. 6). A learning concept is accessible to learn if all its prerequisites are mastered by the learner. Elsewhere, the learning concept is not accessible to learn and no learning resources are attached to it. A concept without prerequisites is always accessible. In our organizer, we distinguish also between a mastered concept and not yet mastered concept. Graphical icons are used to represent the difference between these three learning concept states. For instance, fig. 6 presents an ACM where the learning concept Operator is mastered, the learning concept Procedure is not mastered and the learning concept SemanticLanguage is not accessible. The state of a learning concept can change from inaccessible state to accessible state if its prerequisites become mastered. Also, it can change from non mastered state to mastered state. These alterations in the ACM result from updating the learner model.

\section{Adaptive Course Content}

In our work, learning resources are files with different formats (.pdf, .doc, .html, etc.) queried from web repositories. In our prototype, we use the ARIADNE Knowledge Pool System (ARIADNE KPS), a distributed database of learning resources annotated with LOM metadata elements. In the learning organizer, we propose: (1) A conceptual annotation process for annotating learning resources and (2) a query component for querying learning resources from the local repository. 


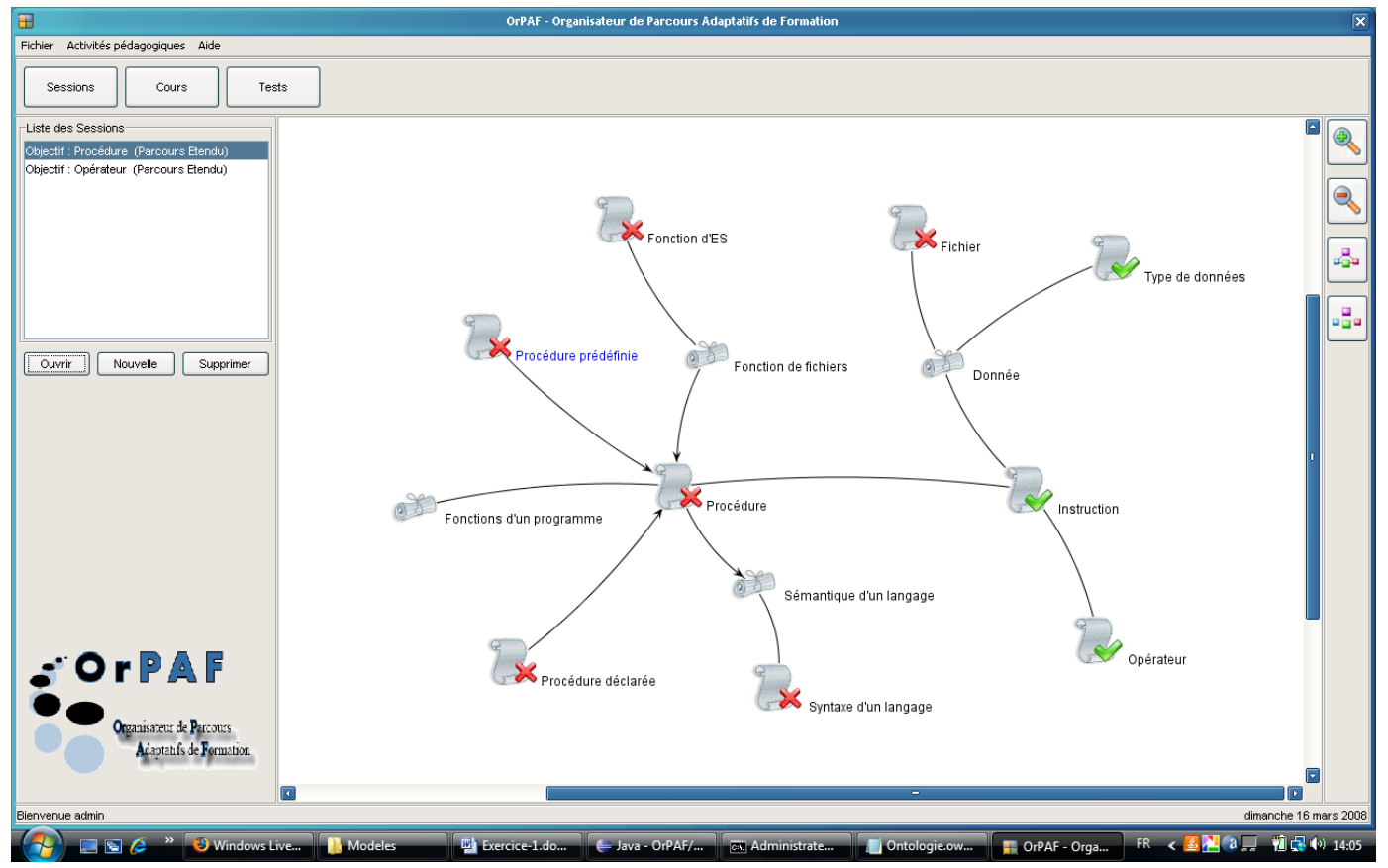

Fig. 6. A screenshot of course structure

\section{Annotating Learning Resources}

Once a distant learning resource is downloaded, it is submitted to a semi-automatic annotation process assisted by a teacher/expert; and finally the learning resource is stored in a local repository. Conceptual annotations of the learning resource are constructed by instantiating some classes and properties from both the domain model and the pedagogical model. For instance, in fig. 7, the learning resource R1 is an Exercise (defined as PedagogicalActivity in the pedagogical model) and teaches the learning topic ComposedStatement (defined as a LearningConcept in the domain model). Characteristics (e.g. learningResourceTopic, learningResourceRole, learningResourceAuthor) of the resource are manually identified by experts and annotations are automatically generated by the system according to learning models. The so-built conceptual annotations are then added to learning resource metadata (in our case, the RDF-LOM binding metadata). 


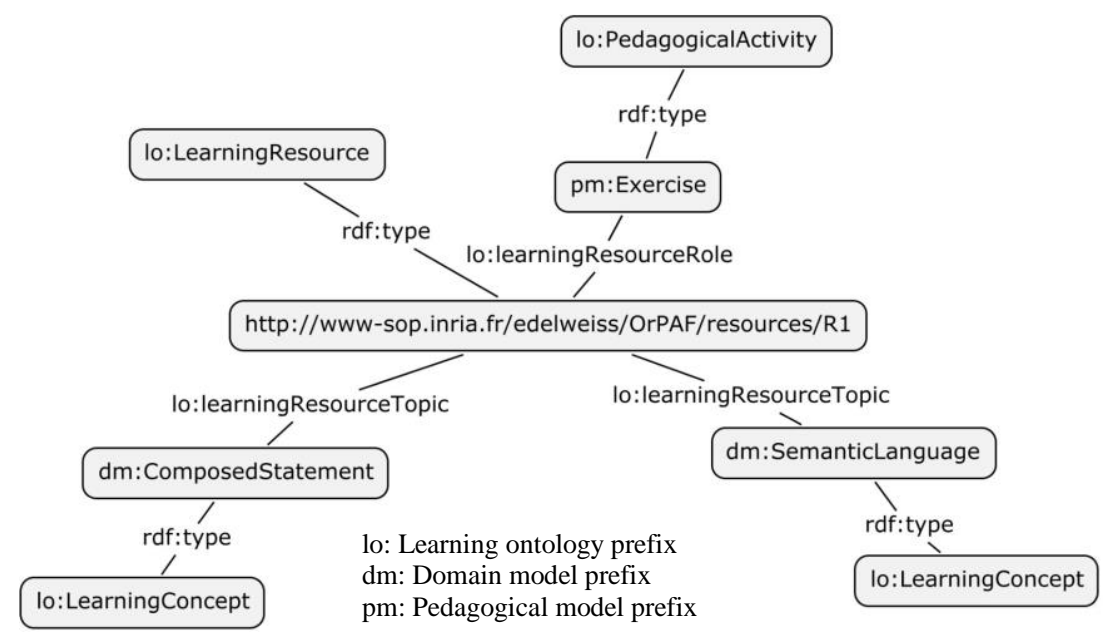

Fig. 7. An excerpt of the conceptual annotation of a learning resource R1

\section{Querying a Local Learning Resource}

The learner can consult resources about accessible learning concepts in his/her ACM by a simple click on it. The local querying component searches for relevant learning resources from the local repository. It computes the relevance of a learning resource by matching its conceptual annotations with the ACM and recommends learning resources to the learner in decreasing order of relevance.

We propose an approach for computing the semantic relevance of a learning resource in a particular learning context. In our case, the learning context is composed of the current learning concept (the concept clicked by the learner) and the state of the ACM. Only learning concepts of conceptual annotations are used in the calculus of the semantic relevance. Our calculus relies on the assignment of a relative weight to each learning concept related to the learning resource. These relative weights depend on the current concept in the ACM and the type of relationships that connect the current concept to concepts related to the learning resource.

Let $\mathrm{P}<\mathrm{c}_{1}, \mathrm{c}_{2}, \mathrm{c}_{3}, . . \mathrm{c}_{\mathrm{n}}>$ be a learning path of length $\mathrm{n}$ and composed of concepts $\mathrm{c}_{\mathrm{i}}$. Let $\mathrm{w}_{\mathrm{ci} / \mathrm{c} 1}$ (i>1) be the weight of concept $c_{i}$ relative to the current $c_{1}$ concept in the ACM. Let $\mathrm{wc}_{1}$ be the weight of the current concept $c_{1}$. We define the relative weight as follows:

$$
\mathrm{w}_{\mathrm{ci} / \mathrm{c} 1}=(1 / \mathrm{a}) \mathrm{w}_{\mathrm{ci}-1 / \mathrm{c} 1}
$$

where $\mathrm{i}>1, \mathrm{w}_{\mathrm{c} 1 / \mathrm{c} 1}=\mathrm{w}_{\mathrm{cl}}=\mathrm{N}$ ( with $\mathrm{N}$ a maximal number) and $\mathrm{a}$ is a variable whose value is as follows:

$\mathrm{a}=2$ (if the relationship between $\mathrm{c}_{\mathrm{i}-1}$ and $\mathrm{c}_{\mathrm{i}}$ is subClassOf or the inverse relationship)

$\mathrm{a}=3$ (if the relationship between $\mathrm{c}_{\mathrm{i}-1}$ and $\mathrm{c}_{\mathrm{i}}$ is prerequisiteOf or the inverse relationship)

$a=5$ (if the relationship between $c_{i-1}$ and $c_{i}$ is aggregationOf or the inverse relationship), etc. 
When there are several relative weights for one learning concept (due to graph cycles) we take the smallest value. Once defined the relative weight of each learning concept related to the learning resource, the semantic relevance SR of the learning resource can be measured as follows:

Let $\mathrm{E}$ be the set of concepts present in the learning resource annotations and present in accessible concepts of the ACM, let F be the set of concepts present in the learning resource annotations and not present in accessible concepts of the ACM, and let $\mathrm{c}$ be the current concept of the ACM.

$$
\mathrm{SR}=\frac{\sum_{x \in E} w_{x / c}}{1+\sum_{y \in F} w_{y / c}}
$$

The definition of SR reflects the fact that the weight of a concept depends on the current concept and the state of the ACM and therefore of the learning context. A resource is relevant if its learning concepts have important relative weights and are largely similar to the accessible learning concepts of the ACM. Otherwise, the resource is less or not relevant.

\section{IMPLEMENTATION AND EVALUATION}

\section{Implementation}

The implemented prototype of the learning organizer integrates several functions to fulfill requirements for the generation of adaptive courses. We used JAVA language for all user interfaces and functions in OrPAF. The interoperability between the implemented prototype and ARIADNE KPS is implemented by a java API named KPS client package. The learning ontology was described in OWL Lite language and learning models were described in RDF language. OrPAF uses Corese, an ontology-based search engine for the semantic web, dedicated to the query of RDF annotations by using the SPARQL query language (Corby et al., 2006). Corese is used to extract concepts and properties of learning models and conceptual annotations. OrPAF prototype is experimented to teach learning concepts of the domain «Algorithmic and programming languages» on a group of thirty learners in graduation of mathematics and computer sciences.

Each learner must give identification information (username and password) in order to access to OrPAF. Once logged in, he/her can:

- Open a new learning session by submitting his/her learning goal concept and his/her time constraints that define the filter type and therefore the size of the ACM.

- Save a current learning session (the ACM, the learner comments, queried learning resources, etc.). Sessions of a learner are stored in his/her learner model.

- Reload an interrupted session. 


\section{Evaluation}

We experimented OrPAF on a group of thirty learners who belong to three different graduation levels. We applied an accurate evaluation protocol: we divided the group into two subgroups A and B. In the first step of the protocol, the learners of group A used the OrPAF whereas the learners of group B used paper-support resources. In the second step of the protocol, the learners of group B were invited to use OrPAF. After each step, an exercise was performed by learners of both groups. It aimed to detect the conceptualization capabilities of learners before and after the use of OrPAF.

The purpose of this evaluation protocol was to analyze behavior /judgments of learners according to three orthogonal directions: the usability of OrPAF, the conceptualization capabilities acquired by learners and the learners' judgments about the relevance of learning resources recommended by OrPAF. For each direction interviews were presented to learners in order to capture their feedback.

Fig. 8 presents synthetic results which reflect global positive judgments of learners about the usability and the utility of OrPAF. The learners of group B appreciated OrPAF more than those of group A - certainly because they made a comparison between learning without and with the learning organizer. They found more assistance with OrPAF. After the step1, learners of group A obtained best results in the exercise of the conceptualization whereas learners of group B had difficulties to perform the exercise and obtained bad results. After the step 2, learners of group B obtained best results in the exercise of the conceptualization. Other experimentation is currently held for evaluating our relevance measure but it is out of the scope of this paper.

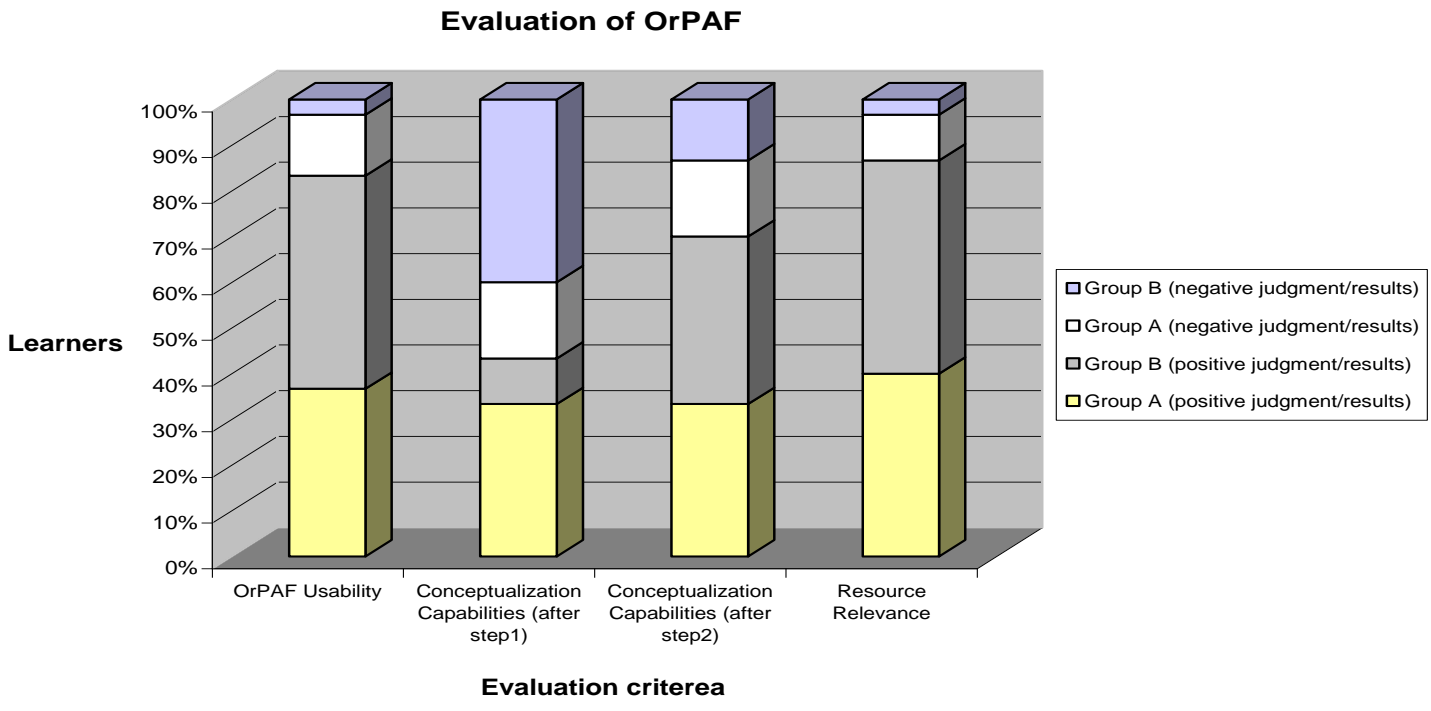

Fig. 8. Results of the experimentation of OrPAF on learners 


\section{CONCLUSION}

This paper presents OrPAF, a learning organizer for the generation of adaptive hypermedia courses which enables a self-regulated learning for learners with different profiles. OrPAF reuses learning resources from distant web repositories. A learning ontology is proposed to describe, in a meta-level, abstract characteristics of an e-learning system. This learning ontology is instantiated to construct a domain model, a learner model and a pedagogical model which describe concrete characteristics of e-learning systems. As a result, the learning organizer is multi-domain and multi-learner profile with minimum changes in the learning models, and the common description of all learning characteristics in the learning ontology improve the reusability of external resources. In contrast with e-learning systems, cited in section 2, the learning organizer provides hypermedia course adaptation for both course structure and course content; and it is an efficient personalization support with few means.

OrPAF generates a hypermedia course as the combination of an adaptive structure and an adaptive content. On the one hand, the course structure is a map of relevant learning concepts. This map is generated by filtering the domain model and applying two layers of adaptation: (1) a first adaptation according to the learning goal and the temporal constraints of the learner; and (2) a second adaptation according to the knowledge of the learner by annotating the concepts of the map. On the other hand, an adaptive content consists of relevant learning web resources. We propose a measure of the semantic relevance of a learning resource for a specific learning context. It is based on relative weights of learning concepts related to the learning resource. These relative weights depend on the learning context.

Experiences with OrPAF led to positive results, both for the usability and the utility of the learning organizer. In addition, we concluded that concept-based course structure develop conceptualization capabilities of learners.

\section{REFERENCES}

Aroyo, L., \& Dicheva, D. (2001). AIMS: Learning and Teaching Support for WWW-based Education. International Journal for Continuing Engineering Education and Life-long Learning, 11 (1/2), 152-164.

Aroyo, L., \& Dicheva, D. (2004). The New Challenges for E-learning: The Educational Semantic Web. Educational Technology \& Society, 7 (4), 59-69.

Brusilovsky, P., Schwartz, E., \& Weber, G. (1996). ELM-ART: An Intelligent Tutoring System on the World Wide Web. In Proceedings of Third International Conference on Intelligent Tutoring Systems (ITS'96), 12-14 June, Montreal, Canada.

Brusilovsky, P., Eklund, J., \& Schwarz, E. (1998). Web-based education for all: a tool for developing adaptive courseware. Computer Networks and ISDN Systems, Proceedings of Seventh International World Wide Web Conference, 291-300.

Brusilovsky, P., \& Vassileva, J. (2003). Course sequencing techniques for large-scale web-based education. International Journal of Continuing Engineering Education and Lifelong Learning 13 (1-2), 75-94.

Corby, O., Dieng-Kuntz, R., \& Faron-Zucker, C. (2004) Querying the Semantic Web with the Corese Search Engine. In Proceedings of ECAI, IOS Press, 705-709.

Corby, O., Dieng-Kuntz, R., Faron-Zucker, C., \& Gandon, F. (2006). Searching the Semantic Web: Approximate Query Processing based on Ontologies. IEEE Intelligent Systems Journal, January/February 2006 (Vol. 21, No. 1). 
De Bra, P., Brusilovsky, P., \& Houben, G.-J. (1999). Adaptive hypermedia: from systems to framework. ACM Computing Surveys, 31 (4es).

De Bra, P., Aerts, A., Berden, B., de Lange, B., Rousseau, B., Santic, T., Smits, D., \& Stash, N. (2003). AHA! The Adaptive Hypermedia Architecture. In Proceedings of fourteenth ACM Conference on Hypertext and Hypermedia, New York: ACM Press, 81-85.

Dehors, S., Faron-Zucker, C., \& Dieng-Kuntz, R. (2006). Reusing Learning Resources based on Semantic Web Technologies. In Proceedings of 6th IEEE International Conference on Advanced Learning Technologies, ICALT 2006, Kerkrade, Netherlands.

Dolog, P., \& Nejdl, W. (2003) Challenges and benefits of the semantic web for user modelling. In Proceedings. of AH2003 - Workshop on Adaptive Hypermedia and Adaptive Web-Based Systems at World Wide Web, UserModelling and Hypertext Conference, (Budapest, Pittsburgh, Nottinngham).

Dolog, P., Henze, N., Nejdl, W., \& Sintek, M. (2004). Personalization in distributed e-learning environments. In Proceedings of Thirteenth International World Wide Web Conference.

Galeev, I., Tararina, L., \& Kolosov, O. (2004). Adaptation on the basis of the skills overlay model. In Proceedings of IEEE International Conference on Advanced Learning Technologies.

Hug, T. (2005). Micro Learning and Narration. In Proceedings of fourth Media in Transition conference, May 68, MIT, Cambridge (MA), USA.

Miklos, Z., Neumann, G., Zdun, U., AND Sintek, M. (2003). Querying semantic web resources using TRIPLE views. In Proceedings of the 2nd International Semantic Web Conference (ISWC2003), Sanibel Island, Florida, USA, Oct.

Murray, T. (2001). Metalinks: authoring and affordances for conceptual and narrative flow in adaptive hyperbooks. The International Journal of Artificial Intelligence in Education.

Nejdl, W., Wolf, B., Qu, C., Decker, S., Sintek, M., Naeve, A., Nilsson, M., Palmer, M., \& Risch, T. (2002). EDUTELLA: a P2P Networking Infrastructure based on RDF. In Proceedings of 11th World Wide Web Conference, Hawaii, USA, May.

Perry, N.E., Phillips, L., \& Hutchinson, L.R. (2004). Preparing student teachers to support for self-regulated learning. Elementary School Journal, 106, 237-254.

Pintrich, P. R., \& Schunk, D. H. (2002). Motivation in education: Theory, research, and applications. (2nd edition). Upper Saddle River, NJ: Merrill, Prentice Hall.

Rada, R., Mili, H., Bicknell, E., \& Blettner, M. (1989). Development and Application of a Metric on Semantic Nets. IEEE Transaction on Systems, Man, and Cybernetics, vol.19(1), 17-30.

Resnik, P. (1995). Semantic Similarity in a Taxonomy: An Information-Based Measure and its Applications to Problems of Ambiguity in Natural Language. Journal of Artificial Intelligence Research, vol 11, 95130.

Ritter, S. (1997). PAT Online: A Model-Tracing Tutor on the World-Wide Web. At the Workshop on Intelligent Educational Systems on the WWW, 18-22 August, Kobe Japan.

Ullrich, C. (2004). Description of an instructional ontology and its application in web services for education. SWEL Workshop, ISWC'2004, Japan, 17-23.

Weber, G., Kuhl, H-C., \& Weibelzahl, S. (2001). Developing adaptive internet based courses with the authoring system netcoach. In Proceedings of third workshop on adaptive hypertext and hypermedia, UM2001, TU/e Computing Science Report 01/11.

Wu, Z., \& Palmer, M. (1994). Verb semantics and lexical selection. In thirty second Annual Meeting of the Associations for Computational Linguistics.

Yessad, A., \& Laskri, M.T. (2006). Ontology-driven Dynamic Course Generation for Web-based Education. In Proceedings of MCSEAI, December, Morocco.

Zhong, J., Zhu, H., Li, J., \& Yu, Y. (2002). Conceptual Graph Matching for Semantic Search. In Proceedings of 10th International Conference on Conceptual Structures, ICCS2002, LNCS 2393, Springer Verlag, 92 106, Borovets, Bulgaria. 